\title{
Criminologie
}

\section{La conduite en état d'ébriété : les impacts des modifications législatives de décembre 1985 tels que perçus par les policiers}

\section{Marie Beaulieu}

Volume 22, numéro 1, 1989

Drogues et criminalités

URI : https://id.erudit.org/iderudit/017272ar

DOI : https://doi.org/10.7202/017272ar

Aller au sommaire du numéro

Éditeur(s)

Les Presses de l'Université de Montréal

ISSN

0316-0041 (imprimé)

1492-1367 (numérique)

Découvrir la revue

Citer cet article

Beaulieu, M. (1989). La conduite en état d'ébriété : les impacts des modifications législatives de décembre 1985 tels que perçus par les policiers. Criminologie, 22(1), 27-40. https://doi.org/10.7202/017272ar
Résumé de l'article

Did the legislative changes of December 1985 concerning impaired driving affect the work of the penal agents? The author answers this question on the basis of interviews with peace officers of the various police corps of Greater Montreal. The Act did change their attitudes as well as their methods of enforcing the legislation. There was also a change in their personal and professional attitudes toward drunken driving. The police say they had noted variations in the attitudes and behaviour of the general public. 


\section{LA CONDUITE EN ÉTAT D'ÉBRIÉTÉ: LES IMPACTS DES MODIFICATIONS LÉGISLATIVES DE DÉCEMBRE 1985 TELS QUE PERÇUS PAR LES POLICIERS \\ Marie Beaulieu*}

Did the legislative changes of December 1985 concerning impaired driving affect the work of the penal agents? The author answers this question on the basis of interviews with peace officers of the various police corps of Greater Montreal. The Act did change their attitudes as well as their methods of enforcing the legislation. There was also a change in their personal and professional attitudes toward drunken driving. The police say they had noted variations in the attitudes and behaviour of the general public.

\section{INTRODUCTION}

En décembre 1985, une modification à la loi régissant la conduite avec les facultés affaiblies apparaissait dans le code criminel canadien. Cette loi modifiée faisait suite à divers travaux, présentés à la demande du gouvernement fédéral, tentant de définir l'alcool et la drogue au volant comme des problèmes sociaux importants au Canada et d'y apporter des solutions. Peu de matériel fut produit sur l'influence des diverses drogues légales et illégales sur la conduite d'un véhicule moteur. Les études ont essentiellement porté sur l'alcool. Ces travaux ont entraîné des modifications des politiques pénales et sociales en matière de conduite sous l'influence d'alcool ou de drogue. (Voir l'article de Pierre Landreville dans la présente livraison). On retrouve le détail de la loi sur la capacité de conduire affaiblie aux articles 237 à 243 du code criminel canadien.

Sans entrer dans tous les détails et les subtilités des modifications légales, on retient que cette législation se distingue des lois précédentes par sa sévérité tant au niveau des sentences (amende et incarcération) que par les interdictions de la conduite d'un véhicule moteur (retrait du permis de conduire). La nouvelle loi facilite le dépistage, donc le travail policier, par la reconnaissance des appareils de détection et la possibilité de prélever un échantillon sanguin, si le conducteur soupçonné d'avoir conduit avec les facultés affaiblies se trouve dans I'incapacité de fournir un échantillon d'haleine suffisant pour l'alcootest.

* L'auteure est agente de recherche au Centre international de criminologie comparée de l'Université de Montréal. C.P. 6128, Succ. A, Montréal, H3C 3J7. 
En réaction aux modifications législatives du code criminel canadien, des altérations furent apportées au code de la route de la province de Québec. Ces changements provinciaux viennent renforcer les sanctions prévues par le fédéral en augmentant, entre autres, la durée du retrait minimum du permis de conduire (par exemple de trois mois à un an pour une première infraction).

\section{NOTRE ÉTUDE}

Nous vivons donc au coeur d'une société ayant défini la conduite avec facultés affaiblies par la drogue ou l'alcool comme un problème social important, nécessitant une intervention accrue de l'État. De la définition de ce problème ont découlé des modifications des politiques sociales et pénales. Mais qu'en est-il de l'intervention des agents pénaux? Les modifications pénales correspondent-elles aux réalités de la pratique pénale? Les agents pénaux ont-ils transformê leur pratique?

Dans le but de répondre aux questions ci-haut mentionnées, nous* avons décidé d'interviewer quarante agents pénaux du Grand Montréal sur leurs attitudes face à la nouvelle loi de décembre 1985, leurs pratiques pénales en matière de conduite en état d'ébriété et leurs évaluations des impacts des modifications législatives. Ces professionnels sont policiers, avocats de la défense, avocats de la couronne, juges, commissaires des libérations conditionnelles, agent de probation et de libération conditionnelle, intervenant en toxicomanie, représentant de la Régie de l'assurance automobile du Québec (RAAQ) et témoins experts du laboratoire médico-légal. Sans entrer dans un débat méthodologique profond, il est entendu que les chercheurs prennent en considération le fait que le discours des intervenants sur leurs pratiques peut grandement différer de la réalité de leurs interventions. Il s'avère impossible de rendre compte justement et précisément des propos de chaque groupe des intervenants pénaux dans un court article. Nous avons donc choisi de nous attarder plus en profondeur à un seul groupe : les policiers. Ce choix, arbitraire nous en convenons, s'explique par le fait que ce sont les policiers qui détiennent les clés de l'entrée ou non d'un cas de conduite avec facultés affaiblies dans les rouages du système pénal.

Avant de répondre à nos interrogations, voici un aperçu de la littérature importante dans le domaine de la conduite en état d'ébriété.

* Marie Beaulieu M.Sc., Mylène Jaccoud M.Sc. et Yves Brillon Ph.D. du Centre international de criminologie comparée de l'Université de Montréal. 


\section{BRÈVE REVUE DE LA LITTÉRATURE}

Une incursion dans la revue de la littérature relative à la conduite en état d'ébriété démontre rapidement que la lutte contre ce mode de conduite ne peut s'effectuer en faisant uniquement appel à des mesures «punitives» ou "répressives». Et cela, malgré le fait que la conduite avec facultés affaiblies suscite de vives réactions au sein des populations.

En effet, depuis quelques années, il semble qu'il y ait eu une évolution des normes et des valeurs touchant la conduite en état d'ébriété, laquelle est maintenant considérée comme plus grave par la population en général. Une recherche auprès du public, réalisée par le Centre international de criminologie comparée de l'Université de Montréal, démontre que la conduite dangereuse (avec mort d'homme) arrive, sur une échelle de gravité, au quatrième rang d'une vingtaine de crimes: cela après l'homicide lors d'un vol à main armée, après le meurtre passionnel, et après le viol accompagné de blessures graves. (Tremblay et al, fév. 1986; texte inédit). Cette évolution de l'opinion publique vue par divers groupes de pression, n'est pas étrangère aux tendances, dans divers pays, d'augmenter les sanctions pénales envers les conducteurs qui, à cause de facultés affaiblies, conduisent leur voiture et sont responsables d'accidents. Or, selon monsieur Donelson, toutes ces lois répressives ne donnent rien. (Gouvernement Fédéral, 1985: p. 13). D'après lui, le problème doit être traité sur le plan des attitudes plutôt que sur celui de la répression: «Je pense qu'avant que les statistiques ne changent de manière appréciable, il faudrait qu'il y ait des modifications au niveau des attitudes sociales et des normes de comportement.» (idem, p. 16).

Les recherches les plus élaborées, faites dans les pays scandinaves (Ross, 1975 a, 1975b; Tokaia, 1978; Votey, 1982 ...), en Europe (McLellan, 1975; Ross, 1981 ...) et au Canada (Donelson et al., ministère de la Justice: Conduite avec Facultés Affaiblies; Rapport No 1, No 2, No 3, No 4, et No 5), ont montré que des politiques uniquement «répressives» de la conduite dangereuse ne diminuaient en rien le problème de l'alcool au volant. Cela, tout simplement parce que les probalités d'accident, pour le conducteur fautif, sont très minces: «Il faut reconnaître, déclare le Dr. Donelson, que les chances qu'un conducteur en état d'ébrićté ait un grave accident de la route sont vraiment très minimes. Le risque absolu correspond peut-être à un trajet sur quatrevingt mille. Vous voyez que le risque est très faible... Chaque fois que quatre-vingt mille personnes en état d'ébriété décident de ne pas prendre le volant de leur voiture, un accident de la route est évité.» 
(Gouvernement fédéral, 1985: p. 17) De plus, les recherches canadiennes, conduites par ce chercheur et son équipe, montrent que le conducteur avec facultés affaiblies a peu de chances de se faire arrêter: environ une chance sur deux mille.

D'autres études canadiennes ont montré que les effets dissuasifs des nouvelles législations sont, en général, assez limités. Une recherche de Smart (1972) le démontre, à partir, il est vrai, d'observations assez limitées. Cet auteur a tenté d'évaluer l'effet du contrôle du taux d'alcool dans le sang sur la conduite dangereuse. Pour ce faire, pendant les trois mois qui ont précédé ainsi que pendant les trois mois qui ont suivi l'entrée en vigueur de la loi de décembre 1969, rendant obligatoire le test de l'ivressomètre, il a comptabilisé (de dix-huit à vingt-deux heures) le nombre de voitures qui se trouvaient dans les stationnements de quatre tavernes. Il a pu noter une baisse considérable du nombre de voitures pendant le premier mois; baisse qui s'estompa graduellement dans les mois subséquents. Les travaux de Carr, Goldberg et Farbar (1975) indiquent, eux aussi, le peu d'incidences positives d'une répression plus sévère sur le nombre d'accidents. De même, une étude de Chambers, Robert et Voelker (1976) conclut que les conséquences de dispositions législatives plus répressives sont de courtes durées.

Ceci nous porte à croire que la solution du problème réside dans un changement d'attitudes, sous-jacent à un changement de politiques. D'une certaine façon, il faut - premièrement - augmenter les chances qu'a un conducteur ivre de se «faire prendre» afin de stopper le nombre d'accidents de la route reliés à une consommation abusive d'alcool. L'expérience anglaise, celle du «Road Safety Act» de 1967, semble avoir eu un effet positif en ce sens. Selon Ross (1973), la baisse du nombre de morts et de blessés en Grande-Bretagne, après l'entrée en vigueur de cette loi, est attribuable au fait que moins de conducteurs ont conduit après avoir bu. En fait, on retourne au principe de Beccaria, à savoir que ce n'est pas tant la sévérite de la peine qui importe mais sa certitude. Et Ross en convient: "certainty of punishment can deter socially harmful behavior.» (p. 3). Deuxièment, il faut sensibiliser la population à la gravité des conséquences qui découlent des accidents causés par des conducteurs en état d'ivresse. Or, les mesures dissuasives, si elles peuvent faire peur pendant un moment, ne changent pas à long terme les attitudes à l'égard de la conduite dangereuse. Comme le souligne Donelson: «À l'heure actuelle, le fait de conduire après avoir trop bu est toujours perçu comme une faute morale exigeant une punition, non un traitement.» (Gouvernement fédéral, $1985:$ p. 21). 
Après les changements apportés au Code pénal canadien, en 1975, rendant passible d'un emprisonnement de deux ans toute personne conduisant un véhicule à moteur alors qu'elle est inhabile à conduire, il n'y a pas eu de baisse dans le nombre de victimes d'accidents graves: «Aucun indice ne permet de conclure avec certitude que l'alcool a joué un rôle moins important dans les accidents mortels depuis l'adoption des nouvelles dispositions au Code criminel» (Carr et al, 1975: p. 57). Selon les recherches de Donelson et al. (1985), il n'y a eu, depuis dix ans, au Canada, ni réduction ni augmentation des pourcentages de morts et de blessures attribuables à l'alcool. La sévérité des lois ne semble pas produire l'effet escompté. Si, en Grande-Bretagne, les mesures législatives ont eu quelque succès, c'est parce que des efforts constants ont été déployés, pendant trois ans, non seulement par la police mais également par les fabricants et les vendeurs de boissons alcoolisées, les mass media, l'industrie des assurances et les organisateurs de programmes communautaires.

L'exemple des pays scandinaves a, depuis plusieurs années, mis en évidence la limite des politiques uniquement punitives. L'attribution de peines d'emprisonnement aux chauffeurs ayant un taux illégal d'alcoolémie n'a pas diminué le nombre de conduite en état d'ébriété. De plus, Bonnie (1981) montre qu'il en va de même, a contrario, puisque là où l'on a remplacé les peines d'emprisonnement par des amendes, il n'y a pas eu d'augmentation du nombre d'accidents. Au Canada, comme le soulignent Nadeau et Poupart (1985), après l'introduction de la loi, en 1969, qui établissait à quatre-vingts milligrammes d'alcool par cent millilitres de sang le taux d'imprégnation alcoolique légalement permis, on a tout d'abord constaté une diminution des accidents de la circulation dans lesquels l'alcool était mis en cause. Cependant, comme on l'a déjà mentionné, au bout de quelques mois les taux retrouvaient les niveaux antérieurs à l'adoption de la loi.

Nadeau et Poupart attribuent, quant à eux, cette modification transitoire des comportements à la publicité entourant la mise en vigueur de lois plus sévères: «Tant qu'on brandit les épouvantails, la perception subjective se modifie. Mais dès que les médias cessent de décrire les nouvelles dispositions plus répressives de la loi, les citoyens reviennent à leurs perceptions antérieures devant la faible probabilité d'être appréhendés...» (Le Devoir, mardi 2 avril 1985).

Une recension des principaux écrits semble donc conduire à la conclusion suivante: aucune loi, si sévère soit-elle, n'aura d'influence directe et durable sur la conduite en état d'ébriété si elle ne s'accom- 
pagne pas d'une campagne d'information de la population de façon à entraîner des modifications au niveau des attitudes et des comportements vis-à-vis de l'ivresse au volant.

\section{NOS RÉSULTATS}

\section{LES ATTITUDES DES POLICIERS FACE À LA NOUVELLE LOI RÉGISSANT LA CONDUITE EN ÉTAT D'ÉBRIÉTÉ}

L'arrivée des modifications législatives a soulevé, à première vue, une réaction officielle très favorable de la part des milieux policiers. Par exemple, l'agent Arès et le sergent Mercier exprimaient leur satisfaction: «C'était dû. C'est bienvenu. Cela va faire réfléchir et ça ne pouvait pas arriver dans un meilleur moment de l'année, soit la période des Fêtes. Les gens vont s'apercevoir - plusieurs le savent déjà - que la boisson au volant ça s'en vient sérieux.» (La Tribune de Sherbrooke, le 6 déc. 1985). Les policiers se réjouissaient aussi beaucoup de l'augmentation de leur moyen de dépistage, soit la reconnaissance légale de leurs appareils de détection portatifs ainsi que la possibilité d'obtenir des télé-mandats pour effectuer un test sanguin à toute personne dans l'incapacité de fournir l'échantillon d'haleine nécessaire pour l'alcootest.

Nos entrevues avec les agents de la paix vont dans le même sens: d'une façon générale, les policiers se réjouissent des resserrements législatifs. Bien que l'ensemble se dit satisfait des modifications législatives, les policiers émettent quelques critiques à l'égard du contenu de la loi.

Premièrement, les législateurs devraient voir à ce que les versions française et anglaise du texte de loi concordent. Deuxièment, quelques agents de la paix questionnent le taux d'alcoolémie prévu par la loi. Pour certains, ce taux devrait être aboli pour être remplacé par des mesures de réflexes et de capacité à conduire alors que le conducteur est sous l'influence de l'alcool. En troisième lieu, des policiers critiquent le principe de l'amende minimale en invoquant que trois cents dollars constituent une peine beaucoup plus sévère pour un assisté social que pour un cadre de compagnie. Quatrièmement, une infime proportion des agents de la paix rencontrés demandent à ce que le permis restreint soit réintroduit. Selon eux, les gens sans permis seraient portés à fuir les lieux d'un accident ou encore à conduire avec des documents falsifiés. Cinquièmement, la majeure partie des policiers rencontrés se disent ravis que la loi prévoie une sentence d'incarcération pour les récidivistes, mais ils attendent avec inquiétude que soient mis en place les programmes de traitement (lors de nos entrevues, les 
programmes gouvernementaux n'avaient pas encore été mis sur pied au Québec).

\section{L'APPLICATION DE LA NOUVELLE LOI}

Une étude ontarienne révèle certaines lacunes dans l'application policière de la loi. (Vingilis $e t a l, 1986$ ). On y apprend, entre autres, que les policiers hésitent davantage à arrêter les femmes que les hommes; qu'ils ne prennent pas les empreintes digitales dans $76 \%$ des cas; que les arrestations sont dues à des manifestations de mauvais contrôle du véhicule ou à des infractions au code de la route plutôt qu'à des barrages routiers; que les policiers sont peu enclins à faire respecter la loi car chaque arrestation leur demande entre une heure et demie et trois heures de travail; qu'ils doivent remplir dix à vingt formulaires par arrestation, ce qui décourage les policiers; que plusieurs policiers considèrent l'alcool au volant comme un délit plus «folklorique» que criminel. Les auteurs concluent qu'il doit y avoir une meilleure formation policière dans le domaine de l'alcool au volant car les actions actuelles ont, pour le moment, peu de chances de diminuer la conduite en état d'ébriété.

Nos entrevues avec les policiers du Grand Montréal révèlent que les conducteurs aux facultés affaiblies sont principalement dépistés au hasard d'une patrouille, lorsque les agents de la paix repèrent un véhicule par sa conduite hasardeuse ou à la suite d'une infraction au code de la route. Même s'il existe une grande publicité entourant les risques d'être interceptés lors d'un barrage routier il semble que dans les faits, ces risques sont minimes. L'utilisation de ces barrages pose des problèmes spécifiques. Premièrement, les endroits sont choisis non seulement en fonction du taux d'accidents mais aussi de la sécurité à y installer un barrage. Des lieux propices aux accidents se trouvent ainsi non contrôlés à cause du danger que pourrait y constituer un barrage. De plus, les barrages routiers sont tributaires de la température: par mauvais temps, il s'avére imprudent de modifier la vitesse du trafic. Finalement, à la suite de jurisprudences contradictoires quant à la légalité des barrages, certains corps policiers publicisent le déroulement d'une telle opération dans les médias. Les personnes en état d'ébriété évitent donc d'emprunter une route pouvant comporter des barrages.

Les cas de conduite avec facultés affaiblies se rencontrent principalement au cours de la fin de semaine, en particulier la nuit, et dans la période des Fêtes et celle des vacances estivales. Quelques agents de la paix disent rencontrer une recrudescence de cas lorsque les prestataires de bien-être social reçoivent leur chèque. 
S'ils ont des motifs de croire que la personne est en état d'ébriété, les policiers peuvent lui demander de se soumettre à des tests symptomatiques ou encore, de souffler dans un dépisteur légalement reconnu. Il semblerait que ces appareils soient en nombre limité, ce qui pose problème lors de l'arrestation. À la Sûreté du Québec (SQ), il y aurait un appareil de disponible pour deux voitures. Par contre, au Service de police de la communauté urbaine de Montréal (SPCUM) seules les équipes chargées de la sécurité routière en seraient munies. En banlieue, rares seraient les corps policiers munis de cet équipement. Les policiers déclarent que s'ils doivent recourir à des tests symptomatiques pour recueillir la preuve, ils seront beaucoup plus interrogés et contreinterrogés en cour. Ils doivent présenter un dossier comprenant de multiples détails permettant de démontrer que le conducteur affichait un taux d'alcool supérieur à la limite légale permise.

Dans les cas où le conducteur est incapable de fournir un échantillon d'haleine, les policiers peuvent avoir recours au télé-mandat pour obtenir la permission de faire faire une prise de sang au conducteur soupçonné d'avoir conduit avec les facultés affaiblies. Les policiers se disent satisfaits que la loi prévoie l'utilisation du télé-mandat mais ils déplorent certains problèmes d'application qu'ils attribuent à un manque de collaboration des juges (beaucoup de questions avant d'accorder un mandat ou refus de la part du juge d'autoriser le mandat) et du personnel médical (lenteur à exécuter la demande, non disponibilité pour collaborer avec la police).

Quand un conducteur est intercepté pour facultés affaiblies, les policiers sont censés mettre en branle tout le processus judiciaire. Cependant, comme dans plusieurs autres arrestations, les agents de la paix peuvent jouir de leur pouvoir discrétionnaire. Certains vont décider de fermer les yeux pour laisser une chance au conducteur, d'autres parce qu'ils ne considèrent pas l'alcool au volant comme une infraction grave, d'autres encore parce qu'ils désirent investir leur temps ailleurs, et finalement, certains agissent ainsi pour s'éviter tout le travail nécessaire (multiples documents à compléter) pour acheminer un dossier devant le tribunal.

S'ils décident de poursuivre leurs démarches, les policiers doivent sommer le conducteur de les accompagner au poste de police afin de subir un test d'ivressomètre. Nous avons noté que les ivressomètres ne sont pas disponibles dans tous les postes de police de la région du Grand Montréal. La plupart des villes de banlieue n'en possèdent pas et 
comptent sur les postes de la SQ. Le SPCUM a choisi d'en placer quatre en des lieux stratégiques : un dans chaque secteur de la ville. $\mathrm{Ce}$ n'est qu'à la SQ que chacun des postes serait doté d'un ivressomètre. Seul un technicien qualifié peut manoeuvrer les appareils. La SQ dit qu'un policier sur deux a obtenu ces qualifications. Au SPCUM, le technicien préposé à l'ivressomètre n'occupe que cette fonction: il cesse d'être patrouilleur. Seuls quelques policiers ont reçu la formation. Il semble qu'à certains moments, le SPCUM, n'a pu offrir le service de l'ivressomètre, faute de personnel qualifié disponible. Après les deux mesures réglementaires à l'ivressomètre, la personne n'ayant pas un taux d'alcoolémie supérieur à la limite permise est relâchée. Pour les autres, la procédure varie d'un service policier à un autre. Au SPCUM, la personne est gardée en cellule jusqu'à ce que ses facultés soient rétablies. À la SQ, les policiers laissent partir le conducteur mais prennent soin de garder son véhicule. En banlieue, il arrive que le conducteur puisse profiter des services d'un agent de la paix pour le conduire à son domicile.

Lors de la préparation du dossier, certains agents de la paix mentionnent qu'ils ont recours à un examen des antécédents de la personne arrêtée à l'aide d'une centrale de renseignements accessible par un ordinateur situé au poste. C'est à partir de ces informations que l'avis de récidive serait déposé au dossier. Cependant, plusieurs des agents de la paix rencontrés mettent en doute la qualité des informations ainsi obtenues. Certains postes de banlieue ne seraient pas équipés de ce système informatique, donc ne nourrissent pas et n'ont pas accès à l'information. L'avis de récidive ne sera déposé que si la dernière infraction en semblable matière a eu lieu dans les derniers mois. À la $\mathrm{SQ}$, on dit considérer les douze derniers mois alors qu'en banlieue, les policiers parlent de vingt-quatre mois. Le dépôt au dossier d'un avis de récidive apparait donc comme tributaire de plusieurs facteurs.

Plusieurs agents de la paix se plaignent de la lourdeur des dossiers de facultés affaiblies. Ils doivent investir beaucoup de temps et rédiger plusieurs documents pour bien monter leur preuve. Ils demandent que les procédures soient revisées afin d'alléger le processus.

Finalement, les policiers se prononcent sur les tribunaux. Plusieurs demandent que soit mise en place une cour où le personnel serait spécialisé dans les infractions reliées à la conduite avec facultés affaiblies. Ils demandent aussi aux juges d'accorder une plus grande importance aux preuves de tests symptomatiques et de cesser de croire en la quasi infaillibilité des preuves par ivressomètre. 


\section{LES IMPACTS DES MODIFICATIONS LÉGISLATIVES}

Selon les policiers rencontrés, les impacts des modifications législatives sont de deux ordres: un premier impact touche l'attitude et les pratiques des policiers à l'égard des cas de conduite avec facultés affaiblies, le second apporte des changements chez le public en général.

\section{A) IMPACTS CHEZ LES POLICIERS}

Selon plusieurs policiers, certains de leurs collègues sont devenus sensibles aux dangers de l'alcool au volant à la suite des changements législatifs. Ils auraient été frappés d'un regain d'énergie dans ces dossiers. Bien entendu, l'impact n'est pas aussi fort pour l'ensemble des policiers. Différentes raisons peuvent amener les policiers à ne pas vouloir s'impliquer. Les deux motifs les plus souvent mentionnés sont le dédain des conducteurs ivres et un problème personnel de consommation excessive d'alcool. Une revue de la littérature de Dietrich et Smith (1986) sur la consommation d'alcool et de drogue chez les policiers affirme que cinq à trente pour cent des policiers abusent d'alcool. Nous ignorons quel est le pourcentage de policiers du Grand Montréal surconsommant ainsi, mais nous croyons qu'il serait des plus intéressant de réaliser une étude sur le lien entre la consommation d'alcool personnelle et l'application de la loi.

En terme de quantité de travail investi de la part des policiers, il est difficile de savoir si la modification législative a eu un impact. Selon certains policiers, il y a eu une augmentation du nombre de dossiers, pour d'autres il n'y a pas de variation. Une chose apparaît certaine pour tous : les policiers ont consacré plus de temps et d'énergie à ces infractions. Des projets d'actions concrètes de dépistage furent réalisés, ce qui a pu entraîner une variation dans le nombre de plaintes officiellement enregistrées.

Les policiers notent, lorsqu'ils se présentent devant les tribunaux, que le changement législatif a entraîné une augmentation du nombre de causes contestées et le développement d'une foule de défenses.

\section{B) IMPACTS AU SEIN DU PUBLIC}

Dans le public en général, les policiers constatent que les modifications législatives ont eu un impact important. Cet impact n'est pas étranger à toute la publicité qui a entouré l'événement. Les policiers sont unanimes pour affirmer que les gens savent qu'il existe une loi sévère s'ils décident de conduire après avoir consommé une certaine quantité de boissons alcoolisées. 
Même s'il semble y avoir une conscientisation du public, il y a toujours des gens qui continuent de conduire après avoir consommé plus d'alcool que la loi ne leur permet. Pour certains, il s'agit d'un geste volontaire, par refus de changer des habitudes. Pour d'autres, il s'agit d'ignorance: ils savent qu'ils ne doivent pas avoir plus de quatrevingts milligrammes d'alcool par cent millilitres de sang mais ils ignorent comment cette mesure se traduit en nombre de consommations.

Un autre impact au sein du public serait la mobilisation d'organismes communautaires autour de la conduite en état d'ébriété. Des efforts de clubs sportifs, d'agences de taxi et autres permettent à des gens en état d'ébriétê d'entrer chez eux rapidement sans conduire leur véhicule moteur.

En terminant, plusieurs policiers demandent que soient poursuivies les campagnes de publicité dans des buts d'éducation du public. Sans déprécier la valeur de la sanction, ils proposent que le gouvernement mise beaucoup plus sur des changements pouvant affecter «l'image» sociale que l'on se fait de la consommation d'alcool. Ils demandent des campagnes réparties sur l'ensemble de l'année (plutôt que concentrées sur certaines périodes comme c'est actuellement le cas), des contenus centrés sur la quantité d'alcool pouvant être consommée qui varie selon chaque personne. Ils insistent principalement sur un programme d'éducation auprès des jeunes, afin de les sensibiliser avant qu'ils n'aient pris des habitudes de consommation d'alcool. Certains critiquent les gouvernements qui acceptent que la publicité associe consommation d'alcool et plaisir et, qu'en même temps, ils prétendent éduquer les gens à contrôler leur consommation ou à prendre leurs responsabilités quand vient le temps de conduire. Il semble y avoir là, dans les politiques gouvernementales, une contradiction flagrante.

\section{COMMENTAIRES}

Comme nous venons de l'illustrer à partir des propos de policiers, il ne fait aucun doute que les modifications législatives de décembre 1985 en matière d'alcool au volant ont eu des répercussions chez les divers agents du système de justice pénal au Canada, tant au niveau des attitudes que des comportements professionnels.

L'attitude générale des policiers face à la nouvelle loi est positive, malgré quelques critiques sur les textes de loi et les principes qui les sous-tendent. 
En terme de comportements, les modifications législatives ont apporté des changements dans la pratique policière. Certaines questions se soulèvent et des problèmes se posent quand on constate qu'il y a des variations importantes dans l'application de la loi peuvant être attribuables aux attitudes et comportements personnels d'un policier ou encore, aux pratiques d'un corps policier par rapport à celles d'un autre. À la lumière des informations que nous avons recueillies, on peut conclure que certains conducteurs ayant un taux d'alcoolémie supérieur au taux légal ont plus de chances de s'en tirer selon le policier qui l'interpelle et son appartenance à un corps policier spécifique.

Finalement, les modifications législatives sont surtout reconnues comme ayant eu de l'impact dans le grand public qui connait maintenant mieux les dangers associés au fait de conduire avec les facultés affaiblies. L'éducation publique en ce domaine doit se poursuivre afin que chaque conducteur connaisse sa limite légale personnelle.

Une étude longitudinale serait souhaitable et nécessaire afin d'évaluer si les impacts des modifications législatives sont circonstanciels ou s'ils s'atténueront avec les années.

\section{RÉFÉRENCES}

BEAULIEU, M.; M. JACCOUD et Y. BRILLON (1988), Les attitudes des agents pénaux face à la législation dans le domaine de la conduite routière. Centre international de criminologie comparée et Centre de recherche en droit public. Université de Montréal.

BONNIE, R.J. (1981), «Discouraging the Use of Alcohol, Tobacco and Other Drugs: The Effects of Legal Controls and Restrictions» in Advances in Substance Abuse : Behaviour and Biological Research. Greenwich, Conn., Jal Press, pp. 145-185.

BRILLON, Y. et M. BEAULIEU (1986), La conduite dangereuse. Rapport final et projet de recherche présentés au ministère de la Justice du Canada. Centre international de criminologie comparée, Université de Montréal.

CARR, B.R.; H. GOLDBERG and C.M.L. FARBAR (1975), The Canadian Breathalizer Translation: an Inferantial Evaluation, in S. Israelstram and S. Lambert (eds.): Alcohol, Drugs and Traffic Safety. Toronto: Addiction Research Foundation of Ontario. pp. 679-687.

CHAMBERS, L.W; R.S. ROBERTS AND C.C. VOELKER (1976), The Epidemiology of Traffic Accident and The Effect of the 1969 Breathalyser Law in Canada. Accident Analysis and Prevention, 8: pp. 201-206.

DIEFRICH, J.; SMITH, J. (1986), The Non-Medical of Drugs Including Alcohol Among Police Personnel: A Critical Litterature Review. Health Directorate, Ottawa.

DONELSON, A.C. (1985), Conduite avec facultés affaiblies, rapport no 4, Alcool et accidents de la route au Canada: stratégies et priorités futures. Ministère de la Justice, Canada. 
DONELSON, A.C. (1985), Conduite avec facultés affaiblies, rapport no 5, Alcool et accidents de la route au Canada: stratégies et priorités futures, résumé. Ministère de la Justice, Canada.

DONELSON, A.C.; D.V. BEIRNESS and D.R. MAYHEW (1985), Conduite avec facultés affaiblies, rappont no 1, Caractéristiques des conducteurs en état d'ébriété. Ministère de la Justice, Canada.

DONELSON, A.C. and D.J. BEIRNESS (1985), Conduite avec facultés affaiblies, rapport no 2, Questions législatives relatives à la conduite sous l'influence de l'alcool. Ministère de la Justice, Canada.

GOUVERNEMENT FÉDÉRAL (1985), De la fondation de recherches sur les blessures de la route au Canada, M. Alan C. Donelson, chercheur principal. Justice et des questions juridiques. Projet de loi C-I8. Fascicule no 11.

McMELLAN, J.K. (1974), Enforcement of the Law in Great Britain Regarding Motorists Impaired by Drink: Success and Problem, in S. Israelstram and S. Lambert (eds.): Alcohol, Drugs and Traffic Safety Toronto: Addiction Research Foundation of Ontario. pp. 705-709.

NADEAU, L.; POUPART, A. (1985), Le projet de loi fédéral sur la conduite en état d'ébriété : une protection accrue du citoyen? Canadian Journal of Public Health. Vol. 76 , nov./déc. 1975.

ROSS, L.H. (1973), Law, Science and Accidents: The British Road Safety Act of 1967. Journal of Legal Studies, 21 : pp. 1-78.

ROSS, L.H. (1975 a), The Scandinavian Myth: The Effectiveness of Drinking and Driving Legislation in Sweden and Norway. Journal of Legal Studies, IV, 2: pp. 285-310.

ROSS, L.H. (1975 b), The Effectiveness of Dinking-and-Driving Law in Sweden and Great Britain, in S. Israelstam and S. Lambert (eds.) Alcohol, Drugs and Traffic Safety Toronto : Addiction Research Foundation of Ontario. pp. 663-678.

ROSS, L.H. (1975 c), L'efficacité des lois sur l'ivresse au volant en Suède et en GrandeBretagne. Toxicomanie, VIII, 1 : pp. 53-76.

ROSS, L.H. (1981), Deterrence of the Drinking Driver: An International Survey. Washington, D.C. : National Highway Traffic Safety Administration.

ROSS, L.H. (1982 a), Interrupted Time Series of Deterrence of Drinking and Driving, in J. Hagan (ed.). Deterrence Reconsidered, England, Sage Publications. pp. 71-99.

ROSS, L.H. (1982 b), Deterring the Drinking Driver: Legal Policy and Social Control. Lexington, MA : Lexington Books.

ROSS, L.H. (1984), Alcohol Impaired Driving in Norway and Sweden : Another Look at "The Scandinavian Myth". Comment. Law and Policy, 6, pp. 1-37.

ROSS, L.H. (1985), Summary of Topic C. Countermeasures, in Tumer et al. "Alcohol and Highway Safety, Proceedings of the North American Conference on Alcohol and Highway Safety held at the Johns Hopkins Medical Institutions». Journal of Studies on Alcohol. No 10, July.

SMART, R. (1972), «Observation of Tavem Patrols Before and After the Compulsery Breath Test Law in Canada. Quartely Journal of Alcohol Studies, 33 : pp. 1122-1128.

TAKALA, H. (1978), Drinking-and-Driving in Scandinavia. Finland. Scandinavian Studies in Criminology, 6: pp. 11-19. 
VINGILIS, E. et al. (1986), Police Enforcement Practices and Perceptions of DrinkingDriving Laws. Revue Canadienne de Criminologie. Avril.

VOTEY, H. (1982), Scandinavian Drinking-Driving Control: Myth or Intuition. The Journal of Legal Studies, XI, 1 : pp. 93-116. 